\title{
Towards Individualized HIV Therapy: Pharmacogenetic-guided Clinical Trials are Needed Now
}

\section{Awewura Kwara*}

Warren Alpert Medical School of Brown University, The Miriam Hospital, 164 Summit Avenue, Providence, RI 02906, USA

Human immunodeficiency virus (HIV) infection is considered a chronic disease that requires life-long suppressive antiretroviral therapy (ART). Current ART is complicated by inter-individual variability in efficacy and toxicity [1]. A systematic review of virologic suppression rate in over 14,000 patients from multiple clinical trials revealed that only $55 \%$ achieved viral loads of $<50$ copies per $\mathrm{mL}$ by week 48 of treatment. Furthermore, up to $45 \%$ of HIV-infected patients discontinue ART during the first year of treatment, with about $51 \%$ of patient changing therapy because of intolerance [2]. The interindividual variability in ART outcomes is due in part to variable antiretroviral drug pharmacokinetics [3]. For some antiretrovirals (ARVs), genetic polymorphisms in drug metabolizing enzymes or transporters contribute to the interindividual variability in pharmacokinetics and response [4]. The current approach to determining effective regimens as well as appropriate dosages of the ARVs through randomized controlled clinical trials identifies effective therapies for the average patient. However, this approach fails to identify effective dosages or therapies for patients who are outliers on dose-response curve. The field of pharmacogenomics is aimed at improving treatment responses using molecular predictors of drug response to individualize regimens or dosingin order to maximize therapeutic benefit while minimizing harm.

Pharmacogenetic research of HIV therapy has generated a large body of literature on genetic predictors of antiretroviral drug pharmacokinetics and drug response [4]. Thisresearch has focused on understanding the relationship between pharmacokinetics and adverse drug reactions and finding genomic markers that can identify individuals at risk of toxicities. Given the complex and polygenic factors that may influence HIV treatment efficacy and toxicities, it is likely that only a limited number of pharmacogenetic association reported in the literature are clinically relevant. Thus, there is the need to clearly demonstrate the clinical utility of the genetic tests for ART prescribing through randomized, pharmacogenetic-guided controlled clinical trials.

The question is when will the accumulated pharmacogenetic knowledge for current ARVs be translated into clinical care of patients? It remains unclear, as there appears to be reluctance in taking the next steps to establish the clinical utility of genetic tests forprescribing potential key candidates. The abacavir story set a clear precedent towards a more effective and safer individualized therapy in HIV care.Abacavir, anucleoside reverse transcriptase inhibitor used in combination regimens for HIV therapy but is associated with severe hypersensitivity reaction in 5-8\% of Caucasians. Following the discovery that HLA-B*5701 gene variant is associated with hypersensitivity reactions to abacavir [5,6], the utility of HLA-B*5701 screening to reduce the risk of hypersensitivity reaction to abacavir was clearly demonstrated in a double-blind, prospective randomized study involving 1956 patients from 19 countries [7]. The results of this landmark study led to incorporation of genetic testing before prescribing abacavir at least in the United States and Europe. What is the next candidate ARV for genomic-driven prescribing? Are we ready for the necessary pharmacogenetic-guided trial?

Currently, there is extensive data on efavirenz pharmacogentics to warrant a genomic-guided clinical trial, with a view of reducing the risk for treatment-limiting toxicities as well as virologic failure. Efavirenz is an essential component of preferred antiretroviral regimens. Efavirenz has a narrow therapeutic index and large interindividual variation in pharmacokinetics and clinical toxicity [8]. Clinically, mid-dose or trough efavirenz plasma concentrations below $1000 \mathrm{ng} / \mathrm{mL}$ has been associated with increased risk of virologic failure, while concentrations above $4000 \mathrm{ng} / \mathrm{mL}$ have been associated with risk of central nervous system side effects [8]. Nearly a third of patients who initiated efavirenz in the Swiss HIV cohort between 2004 and 2007 discontinued the drug in the first year [9]. Is it possible that most of the treatment discontinuation could have been avoided if patients at high risk for toxic plasma concentrations of efavirenz received alternate therapy or were given a lower dose of efavirenz based on genetic tests? We will never know until pharmacogenomics-guided controlled trials are trials are undertaken.

CYP2B6 516TT genotype is a well-recognized predictor of supratherapeutic concentrations of efavirenz [10]. CYP2B6 516TT genotype has also been associated with CNS symptoms at week one of therapy [11], and persistent sleep disorders [12]. Other polymorphisms in CYP2A6 and UGT2B7 gene influence efavirenz plasma concentration but their effect is minimal compared to $C Y P 2 B 6516 \mathrm{G} \rightarrow \mathrm{T}$ polymorphism [13]. While the relationship between efavirenz concentrations and neuropsychological toxicity is not clearly understood, genotypetailored dosing has been used successfully to reduce the persistent of CNS toxicities [14]. In the design of a pharmacogenetic-guided trial of efavirenz therapy, individuals with slow and extensive metabolizing genotype could be randomized to standard efavirenz therapy or alternate therapy. Alternatively, these patients could be randomized to receive standard or adjusted dosingbased on genotype. Other ARVs with emerging pharmacogenetic data that could potentially be translated into clinical practice include atazanavir, tenofovir, lopinavir/ritonavir

*Corresponding author: Awewura Kwara, Warren Alpert Medical School of Brown University, The Miriam Hospital, 164 Summit Avenue, Providence, RI 02906, USA, Tel: 401 793-2463; Fax: 401 793-4704; E-mail: akwara@lifespan.org

Received January 23, 2012; Accepted January 24, 2012; Published January 26 2012

Citation: Kwara A (2012) Towards Individualized HIV Therapy: Pharmacogeneticguided Clinical Trials are Needed Now. J Pharmacogenom Pharmacoproteomics 3:e116. doi:10.4172/2153-0645.1000e116

Copyright: (c) 2012 Kwara A. This is an open-access article distributed under the terms of the Creative Commons Attribution License, which permits unrestricted use, distribution, and reproduction in any medium, provided the original author and source are credited. 
Citation: Kwara A (2012) Towards Individualized HIV Therapy: Pharmacogenetic-guided Clinical Trials are Needed Now. J Pharmacogenom Pharmacoproteomics 3:e116. doi:10.4172/2153-0645.1000e116

and nevirapine [4]. There is pharmacogenetic data that may explain atazanavir--associated hyperbilirubinemia, tenofovir tubulopathy, lopinavir/ritonavir associated dyslipidemia and nevirapine associated hepatotoxicity [4]. While some of these associations need to be confirmed, it is about time to consider a genomic-guided trial of efavirenz therapy. Genomic-driven prescribing of efavirenz has potential to reduce human sufferingand prolong the durability of first-line regimens by reducing the occurrence of neuropsychological symptoms, and early treatment discontinuation or virologic failure. It may also be cost-effective especially in African and Asian population in whom the frequency of CYP2B6 516TT genotype approaches $25 \%$.

Not withstanding the potential of benefit of pharmacogeneticguided trials to move genetic tests for ART into clinical practice, there are some challenges to trial design [15]. There is currently inadequate data on relationship between genomic markers and/or drugs concentrations with clinical outcomesto inform trial design and power calculations. Also, treatment responses may be influence by several genetic and nongenetic factors as well as modifying effect by concurrent medications. Results of pharmacogenomics trials may be useful in some but not all populations depending on the frequency of the putative genetic polymorphism(s). For example, HLA-B ${ }^{*} 5701$ genotyping appears to be cost effective in white populations but not among Asian and or black African in whom the variant frequency is low. The pharmacogeneticguided trials are expensive and the pharmaceutical industry may be reluctant to fund trials or develop drugs that will require genetic tests for prescribing, as this will lead to restriction in the use of their drugs based on patient genotype. In addition, there may be there may be ethical issues as cost of tests might influence equity and access to effective and safe therapy.

In summary, the development of use genomic tools to increase therapeutic benefit while reducing harm is the future of pharmacotherapy of HIV. The use of genetic markers to predict appropriate drug selection or dosages holds promise for reducing undesirable drug effects such as toxicities or treatment failure through individualized therapy. In my opinion, there is currently adequate genetic association data to inform pharmacogenetic-guided trial of efavirenz.

\section{References}

1. Bartlett JA, Fath MJ, Demasi R, Hermes A, Quinn J, et al. (2006) An updated systematic overview of triple combination therapy in antiretroviral-naive HIVinfected adults. AIDS 20: 2051-2064.

2. Vo TT, Ledergerber B, Keiser O, Hirschel B, Furrer H, et al. (2008) Durability and outcome of initial antiretroviral treatments received during 2000--2005 by patients in the Swiss HIV Cohort Study. J Infect Dis 197: 1685-1694.

3. Fabbiani M, Di Giambenedetto S, Bracciale L, Bacarelli A, Ragazzoni E, et al. (2009) Pharmacokinetic variability of antiretroviral drugs and correlation with virological outcome: 2 years of experience in routine clinical practice. $J$ Antimicrob Chemother 64: 109-117.

4. Telenti A, Zanger UM (2008) Pharmacogenetics of anti-HIV drugs. Annu Rev Pharmacol Toxicol 48: 227-256.

5. Mallal S, Nolan D, Witt C, Masel G, Martin AM, et al. (2002) Association between presence of HLA-B*5701, HLA-DR7, and HLA-DQ3 and hypersensitivity to HIV1 reverse-transcriptase inhibitor abacavir. Lancet 359: 727-732.

6. Hetherington S, Hughes AR, Mosteller M, Shortino D, Baker KL, et al. (2002) Genetic variations in HLA-B region and hypersensitivity reactions to abacavir. Lancet 359: 1121-1122.
7. Mallal S, Phillips E, Carosi G, Molina JM, Workman C, et al. (2008) HLA-B5701 screening for hypersensitivity to abacavir. N Engl J Med 358: 568-579.

8. Marzolini C, Telenti A, Decosterd LA, Greub G, Biollaz J, et al. (2001) Efavirenz plasma levels can predict treatment failure and central nervous system side effects in HIV-1-infected patients. AIDS 15: 71-75.

9. Lubomirov R, Colombo S, di lulio J, Ledergerber B, Martinez R, et al. (2011) Association of pharmacogenetic markers with premature discontinuation of first-line anti-HIV therapy: an observational cohort study. J Infect Dis 203: 246257.

10. Tsuchiya K, Gatanaga H, Tachikawa N, Teruya K, Kikuchi Y, et al. (2004) Homozygous CYP2B6 *6 (Q172H and K262R) correlates with high plasma efavirenz concentrations in HIV-1 patients treated with standard efavirenzcontaining regimens. Biochem Biophys Res Commun 319: 1322-1326.

11. Haas DW, Ribaudo HJ, Kim RB, Tierney C, Wilkinson GR, et al. (2004) Pharmacogenetics of efavirenz and central nervous system side effects: an Adult AIDS Clinical Trials Group study. AIDS 18: 2391-2400.

12. Rotger M, Colombo S, Furrer H, Bleiber G, Buclin T, et al. (2005) Influence of CYP2B6 polymorphism on plasma and intracellular concentrations and toxicity of efavirenz and nevirapine in HIV-infected patients. Pharmacogenet Genomics 15: $1-5$

13. Kwara A, Lartey M, Sagoe KW, Kenu E, Court MH (2009) CYP2B6, CYP2A6 and UGT2B7 genetic polymorphisms are predictors of efavirenz mid-dose concentration in HIV-infected patients. AIDS 23: 2101-2106.

14. Gatanaga H, Hayashida T, Tsuchiya K, Yoshino M, Kuwahara T, et al. (2007) Successful efavirenz dose reduction in HIV type 1-infected individuals with cytochrome P450 2B6 *6 and "26. Clin Infect Dis 45: 1230-1237.

15. Haas DW, Kuritzkes DR, Ritchie MD, Amur S, Gage BF, et al. (2011) Pharmacogenomics of HIV therapy: summary of a workshop sponsored by the National Institute of Allergy and Infectious Diseases. HIV Clin Trials 12: 277 285 